\title{
EDITORIAL
}

\section{Raising the awareness: projecting the future burden of COPD with the BOLD model}

\author{
M. Rutten-van Mölken
}

$\mathbf{N}$ ot so long ago, cost-of-illness studies were criticised for mainly estimating the annual costs of a disease in retrospect, without paying attention to trends in costs and the return on the investments in terms of health benefits. From the time when we began developing models that can simulate the future burden and costs of a disease, we recognised the importance of cost-of-illness studies for providing insight into future healthcare needs and planning purposes. Providing this insight is one of the goals of the Burden of Obstructive Lung Disease (BOLD) project.

The BOLD project aims to measure the prevalence of chronic obstructive pulmonary disease (COPD) among the population aged $\geqslant 40 \mathrm{yrs}$, its risk factors and its impact on quality of life, activity limitation, respiratory symptoms and use of healthcare services in various countries around the world, using a standardised protocol that includes spirometry [1,2]. Among the primary objectives of the BOLD initiative was the development of a COPD health economic model to make projections of the burden of COPD in the future. In the current issue of the European Respiratory Journal, NiELSEN et al. [3] have used this model to estimate the current and future costs of COPD in Iceland and Norway over a 10- and 20-yr period. These are the first cost-of-illness data for COPD in these countries.

The 10-yr costs of COPD were estimated to be $€ 130$ million in Iceland and $€ 1,539$ million in Norway (price level 2005; 3\% discounting). The annual costs of COPD accounted for $1.2 \%$ and $0.7 \%$ of the healthcare budgets in Iceland and Norway. Given that the COPD prevalence, as found in the BOLD survey (17\% in Iceland and 19\% in Norway), is higher than typically reported in previous studies [4, 5], these estimates are surprisingly in line with other countries $[6,7]$. This is because the annual costs per COPD patient were relatively low: $€ 478$ in Iceland and $€ 284$ in Norway. These estimates were low because of the low rates of exacerbations reported in the BOLD survey. The annual rate of moderate exacerbations (requiring contact with a healthcare provider) was only 0.02 in Global Initiative for Chronic Obstructive Lung Disease (GOLD) stage I. In GOLD stage III+ it was 0.13 , considerably smaller

Institute for Medical Technology Assessment, Erasmus University/Erasmus Medical Centre, Rotterdam, The Netherlands.

CORRESPONDENCE: M. Rutten-van Mölken, Institute for Medical Technology Assessment, Erasmus University/Erasmus Medical Centre, P.0. Box 1738, 3000 DR Rotterdam, The Netherlands. E-mail: m.rutten@bmg.eur.nl than in GOLD stage II, where it was 0.33. The rate of severe exacerbations (requiring hospital admission) in GOLD stage III+ was only 0.04 . This may be related to the population-based sampling method, the retrospective nature of the BOLD survey or the length of the recall period in the survey. Had the authors used the lower limit of normal of the forced expiratory volume in $1 \mathrm{~s} /$ forced vital capacity $(\mathrm{FEV} 1 / \mathrm{FVC})$ ratio [8] instead of a fixed FEV1/FVC ratio below 0.7 when diagnosing COPD, the prevalence estimates would have been more conservative and the exacerbation rates would probably have been higher.

This is also the first publication on the BOLD model itself. It is good that the authors restricted the analyses to two countries, because that gave them the space to open-up the black box of the model. If they had included more countries they would not have had the room to explain the structure of the model, the relationship between model parameters and data sources used to estimate parameter values. Opening the black box is crucial to the acceptance of the model results by policy makers.

The BOLD model is a Markov Model, or state transition model [9]. It has nine health states (smoker without COPD, ex-smoker without COPD, never-smoker without COPD, nonsmoker with COPD in GOLD stage I, II or III+, and smoker with COPD in GOLD stage I, II or III+) and a death state. At the start, all subjects in the general population aged $\geqslant 40 \mathrm{yrs}$ are placed in one of the nine health states. In pre-specified time intervals of $1 \mathrm{yr}$ the subjects move through these states. Within the COPD health states subjects can only get worse. Different exacerbation rates, resource use and costs are attached to the health states. The exacerbation rates are divided into mild, moderate and severe exacerbations using resource-use based definitions. Resource use and costs are also attached to the exacerbations.

A model like the BOLD model requires a wide variety of different input parameters. Ideally, input data are needed for each stratum defined by age, sex, smoking status and GOLD stage. Central to the model is the accurate estimation of COPD progression. Like all other COPD models [10], disease progression is rather narrowly defined as the decline in FEV1\% predicted, which drives the probabilities to progress to the next GOLD stage. The BOLD model is the first to use data on lung function decline from the Framingham Heart Study to estimate transition probabilities between GOLD stages [11-13]. Previously published COPD models have either used the US Lung Health Study (LHS) [14-16], the Obstructive Lung Disease in Northern Sweden study (OLIN) [17] or relatively short-term clinical trial data [18]. The advantage of the Framingham Heart Study is that it is population based, 
has a long follow-up, includes patients with COPD from all severity classes, and has data on post-bronchodilator FEV1, just like the LHS. However, valid lung function measurements are not available in all examinations and the number of spirometry tests performed per subject over time is limited. Moreover, a relatively high percentage of COPD patients in the Framingham Heart Study had never smoked, raising the question of whether they really had COPD or a reduced lung function due to other diseases, or a normal lung function with an FEV1/FVC $<0.7$ but still above the lower limit of normality. The LHS is limited by the fact that it is a clinical trial which enrolled patients with mild-to-moderate COPD, but who did not regard themselves as having a lung disease. Thus, it has to be assumed that these data are applicable to those with (very) severe COPD. However, the LHS is a large study, patients are closely monitored with annual spirometry and the impact of smoking cessation and relapse to smoking on lung function decline can be estimated separately. The OLIN data are population based but the transition probabilities have never been published.

The transition probabilities are assumed to be transferable to other countries, outside the USA; they do not have to be changed when using the model in other countries. The BOLD model can easily be filled with country-specific data. The more country-specific data sources the more representative the burden-of-illness estimate. NIELSEN et al. [3] have used country-specific estimates of smoking prevalence, incidence and prevalence of COPD, all-cause mortality, respiratoryspecific mortality, exacerbation rates, resources and costs for maintenance treatment, and resources and costs for exacerbation treatment, although the latter had to be based on expert opinion. COPD prevalence, smoking status among patients with COPD, exacerbation rates (from the two surveys combined) and medication use were obtained from the BOLD surveys. However, filling the model with countryspecific data is more easily said than done. For some input parameters there were no country-specific data available. Especially data on incidence of COPD by GOLD stage are hard to obtain, not only in Iceland and Norway. NIELSEN et al. [3] obtained the overall incidence of COPD, for both Iceland and Norway, from a small Norwegian study in which the cumulative 9-yr incidence of COPD was only 40 [19]. All incident cases were placed in stage I COPD, although the study indicated otherwise. They used relative risks for development of COPD from the Framingham Heart Study to divide the incidence over smoking stages. In addition, smoking cessation and relapse rates had to be obtained from US sources (US National Health Interview Survey and US Surgeon general report), as well as mortality risks (National Health And Nutrition Examination Survey).

NIELSEN et al. [3] have used the BOLD model in a cost-of-illness study. However, the BOLD model can be used to calculate the cost-effectiveness of a particular treatment or intervention by synthesising the evidence regarding the costs and effects of the treatment and its alternatives from difference sources. The current model can perform economic evaluations of treatments that improve disease progression, reduce the frequency/ severity of COPD exacerbations or reduce mortality. However, the impact of treatments slowing down progression may not be fully captured because GOLD stages III and IV are combined into one, i.e. stage III+. Treatments that primarily improve health-related quality of life or exercise performance, such as pulmonary rehabilitation programmes, can only be evaluated when quality of life scores or utilities are attached to the health states. The cost-effectiveness of smoking cessation interventions in COPD cannot be evaluated because former smokers with COPD and never-smokers with COPD are combined into one state, i.e. nonsmokers with COPD. Perhaps the greatest potential value of a model like BOLD is that is can facilitate the transferability of country-specific cost-effectiveness studies to other countries, settings or jurisdictions.

One final thought on the potential improvement of the model. The model is not, or not yet, probabilistic. Probabilistic models are models in which the input parameters are not entered as point estimates but as distributions from which random draws can be made [20]. Technically it is not so difficult to make a model probabilistic, and the developers certainly have the expertise to do so, but it largely increases the data requirements. This implies that, at the moment, the BOLD model, like the other previously published models in COPD, can only present results as point estimates without an indication of the uncertainty surrounding these estimates.

\section{STATEMENT OF INTEREST}

A statement of interest for M. Rutten-van Mölken can be found at www.erj.ersjournals.com/misc/statements.dtl

\section{REFERENCES}

1 Buist AS, Vollmer WM, Sullivan SD, et al. The Burden of Obstructive Lung Disease Initiative (BOLD): rationale and design. COPD 2005; 2: 277-283.

2 Buist AS, McBurnie MA, Vollmer WM, et al. International variation in the prevalence of COPD (the BOLD Study): a population-based prevalence study. Lancet 2007; 370: 741-750.

3 Nielsen R, Johannessen A, Benediktsdottir B, et al. Present and future costs of COPD in Iceland and Norway: results from the BOLD study. Eur Respir J 2009; 34: 850-857.

4 Halbert RJ, Isonaka S, George D, et al. Interpreting COPD prevalence estimates: what is the true burden of disease? Chest 2003; 123: 1684-1692.

5 Halbert RJ, Natoli JL, Gano A, et al. Global burden of COPD systematic review and meta-analysis. Eur Respir J 2006; 28: 523-532.

6 Ruchlin HS, Dasbach EJ. An economic overview of chronic obstructive pulmonary disease. Pharmacoeconomics 2001; 19: 623-642.

7 Hoogendoorn M, Feenstra TL, Rutten-van Molken MP. [Projections of future resource use and the costs of asthma and COPD in the Netherlands]. Ned Tijdschr Geneeskd 2006; 150: $1243-1250$.

8 Swanney MP, Ruppel G, Enright PL, et al. Using the lower limit of normal for the FEV1/FVC ratio reduces the misclassification of airway obstruction. Thorax 2008; 63: 1046-1051.

9 Briggs AH, Sculpher M. An introduction to Markov modelling for economic evaluation. Pharmacoeconomics 1998; 13: 397-409.

10 Rutten-van Mölken MP, Lee TA. Economic modeling in chronic obstructive pulmonary disease. Proc Am Thorac Soc 2006; 3: 630-634.

11 Lee TA, Gao C-Y, Walter B, et al. Rate of disease progression in patients with COPD in the Framingham Heart Study Cohort. Chest Meeting Abstracts 2006; 130: 97S.

12 Dawer TR, Meadors GF, Moore FE, Jr, Epidemiological approaches to heart disease: the Framingham Study. Am J Public Health Nations Health 1951; 41: 279-281. 
13 Kannel WB, Feinleib M, MacNamara PM, et al. An investigation of coronary heart disease in families. The Framingham Offspring Study. Am J Epidemiol 1979; 110: 281-290.

14 Hoogendoorn M, Rutten-van Mölken MP, Hoogenveen RT, et al. A dynamic population model of disease progression in COPD. Eur Respir J 2005; 26: 223-233.

15 Sin DD, Golmohammadi K, Jacobs P. Cost-effectiveness of inhaled corticosteroids for chronic obstructive pulmonary disease according to disease severity. Am J Med 2004; 116: 325-331.

16 Spencer M, Briggs AH, Grossman RF, et al. Development of an economic model to assess the cost effectiveness of treatment interventions for chronic obstructive pulmonary disease. Pharmacoeconomics 2005; 23: 619-637.
17 Borg S, Ericsson A, Wedzicha JA, et al. A computer simulation model of the natural history and economic impact of chronic obstructive pulmonary disease. Value Health 2004; 7: 153-167.

18 Rutten-van Molken MP, Oostenbrink JB, Miravitlles M, et al. Modelling the 5-year cost effectiveness of tiotropium, salmeterol and ipratropium for the treatment of chronic obstructive pulmonary disease in Spain. Eur J Health Econ 2007; 8: 123-135.

19 Johannessen A, Omenaas E, Bakke P, et al. Incidence of GOLDdefined chronic obstructive pulmonary disease in a general adult population. Int J Tuberc Lung Dis 2005; 9: 926-932.

20 Weinstein MC. Recent developments in decision-analytic modelling for economic evaluation. Pharmacoeconomics 2006; 24: 1043-1053. 